\title{
NANOPOROUS-BASED HYDROPHILIC POLYMER MICROCHANNEL USING A SPRAY LAYER-BY-LAYER ELECTROSTATIC NANO-ASSEMBLY
}

\author{
K.K. Lee * and C.H. Ahn \\ Microsystems and BioMEMS Laboratory, \\ School of Electronics and Computing Systems, University of Cincinnati, Cincinnati, Ohio, USA
}

\begin{abstract}
A simple, versatile, cost-effective, and high-throughput spray layer-by-layer (LbL) electrostatic nano-assembly technology has been developed, characterized and applied to polymer microchannels with nanoporous surfaces sprayed on cyclic olefin copolymer (COC) for a capillary micropump or separator. The pumping capabilities achieved from the sprayed nanoporous surfaces were mainly due to the strong hydrophilic properties of the multi-coated bilayers of hydrophilic silica nanoparticles. The hydrophilic nature of nanoporous surface of the COC microchannel was characterized by measuring the length of a test liquid driven by the capillary forces produced through the microchannel. The spray LbL method developed in this work can be an innovative approach for the modification of various surfaces of polymer microchannels.
\end{abstract}

\section{INTRODUCTION}

Flow control of biological samples or reagents using capillary force through a microchannel is very attractive for the on-chip microfluidic devices of biological sensing or diagnostic systems [1, 2]. Specifically, superhydrophilic wetting properties have significant effects on liquid behavior in the surface control applications such as capillary pumping or separator [3]. So, the control of surface energy over the microchannel walls is desirable for enhancing the wetting properties of microchannel surfaces.

Polymer-based microfluidic devices are alternative to those fabricated from glass and silicon substrates along with the standard lithographic microfabrication technology [4]. Polymers are suitable for single-use disposable devices since they offer low cost, good biocompatibility and mass manufacture using high-throughput microfabrication techniques such as hot embossing or injection molding. Various polymer materials such as COC, polyimide, polymethylmethacrylate (PMMA), polydimethylsiloxane (PDMS) and polycarbonate (PC) have been used for the microfluidic devices. Among them, COC is of particular interest due to its combination of excellent UV transparency, low autofluorescence, low oxygen permeability, high mechanical strength, and compatibility with a broad range of chemicals and solvents [5].

However, like most other polymers, COC has a hydrophobic nature, which causes many problems in the applications of biochip or lab chip such as sample loss, degradation of resolution in separations, and difficulties with accurate quantitative analyses. In order to minimize these problems, it is necessary to chemically modify or fuctionalize the walls of the COC microchannels. Various techniques of polymer surface modification have been developed [6-10]. However, most of these procedures are not suitable for microfluidic devices since they rarely allow selective patterning on the surface of microchannel. Furthermore, they require additional special instruments, harsh processing conditions, and high cost. So, there is still a large demand for the development of a new technique for attaining highly hydrophilic surfaces on the patterned COC microchannels.

In this work, a new simple, fast, versatile, effective and functional coating method using a spray layer-by-layer (LbL) nano-assembly has been developed to selectively modify the surface of COC polymer microchannels for various microfluidic devices. The major advantage of this spray LbL is a dramatically reduced processing time needed for the multilayer formation at room temperature. Surface functionality can be easily controlled by choosing appropriate polyelectrolytes.

\section{NANO ASSEMBLY}

An aluminum master mold was fabricated first using a micromilling machine (Microlution 5100S) for the replica of COC microchannels. Then, COC substrates were patterned with microfluidic channels using hot embossing technique for the spray $\mathrm{LbL}$ process. The dimension of fabricated microchannel was 400 $\mu \mathrm{m} \times 200 \mu \mathrm{m} \times 47 \mathrm{~mm}$. Then, the spray LbL nano-assembly process with silica nanoparticles was followed for the surface modification of the patterned COC microchannel.

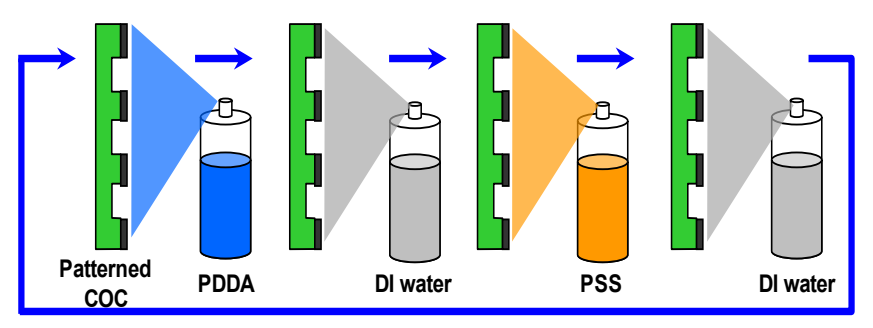

Figure 1: Schematic illustration of spray-LbL nanoassembly of multilayer films on patterned COC microchannels.

Figure 1 shows the conceptual diagram of the spray LbL technology. The surface was modified by successive spraying of poly (dimethyldiallyl-ammonium chloride) (PDDA) and poly (sodium 4-styrenesulfonate) (PSS) (Sigma-Aldrich, St. Louis, MO) as an adhesion promoter on the COC microchannel surfaces, which were already activated by oxygen plasma. Rinsing step was needed after a complete layer was formed. Then, the layer of oppositely charged polyion was sprayed. The polyion solutions and rinsing solutions were supplied by enforced spraying in this process. As hydrophilic nanoparticles with different sizes, SM-30 colloidal silica $30 \mathrm{wt} \%$ (7 $\mathrm{nm}$ diameter silica particles, Sigma-Aldrich), Ludox HS-40 colloidal silica 40 wt \% (12 nm diameter silica particles, Sigma-Aldrich), Ludox TM-40 colloidal silica 40 wt \% (22 nm diameter silica particles, Sigma-Aldrich), and silica nanospheres (5\% aqueous dispersion, $50 \mathrm{~nm} \& 100 \mathrm{~nm}$ diameter silica nanoparticles, Polysciences Inc.) were used for the nanoassembly. A typical multilayer build-up process by spray LbL is schematically described in Figure 2. In Figure 2, 3s, 27s, 20s, and $10 \mathrm{~s}$ indicate the desired processing time in seconds for each process. A polyelectrolyte (PDDA or PSS or silica) was applied by spraying. Spraying is suspended but drainage and evaporation of water continue. The film was spray-rinsed with deionized (DI) water. Then, spray rinsing was suspended but drainage and evaporation of water continue. The total deposition time per layer was 60 seconds. Negatively charged colloidal silica nanoparticles (with average particle sizes of 7, 12, 22, 50, and $100 \mathrm{~nm}$ ) were diluted to 0.3 wt $\%$ in DI water. Polymer solutions (PDDA, PSS) 
were diluted to $0.01 \mathrm{M}$ in DI water.

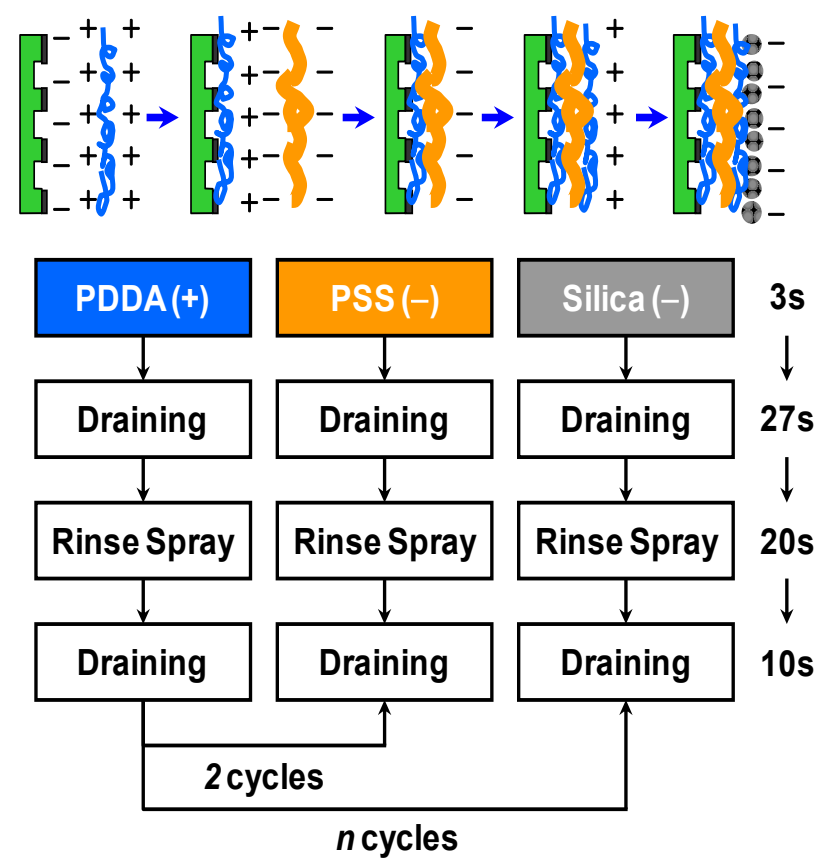

Figure 2: Nano-assembly protocol for the deposition of polyelectrolyte multilayer films on patterned COC microchannels by spray LbL, where $n$ is the number of bilayers. Spraying times and polyelectrolyte concentrations were based on the previous work [11], and no drying step was performed between layer depositions.

\section{EXPERIMENT AND RESULTS}

\section{Contact Angle Measurement}

The wettability of the spray coated surfaces of COC was characterized in ambient air at room temperature using a contact angle analyzer based on the sessile drop method. The mean contact angles are determined by averaging values measured at five different points on the sample surface. In Figure 3, contact angles on the modified COC surfaces were measured as a function of the nanoparticle sizes. The lowest contact angle with smallest standard deviation was obtained in the COC surface coated with 12 $\mathrm{nm}$ silica nanoparticles. So, the $12 \mathrm{~nm}$ silica nanoparticle was chosen for the spray LbL nano-assembly on the COC microchannels as the surface control applications such as capillary pumping or separator.

\section{Surface Characterization with SEM and EDS}

The COC microchannel and the nano silica film formed by silica nanoparticles were characterized by SEM (XL-30, ESEM, Philips) as shown in Figure 4. Silica nanoparticles can be seen in the microchannels and the inset SEM image is a high resolution image of the nanoparticles. These close and uniform microstructures, which contribute to absorb liquid and enhance the nanoporous capillary action, can be observed in the SEM image.

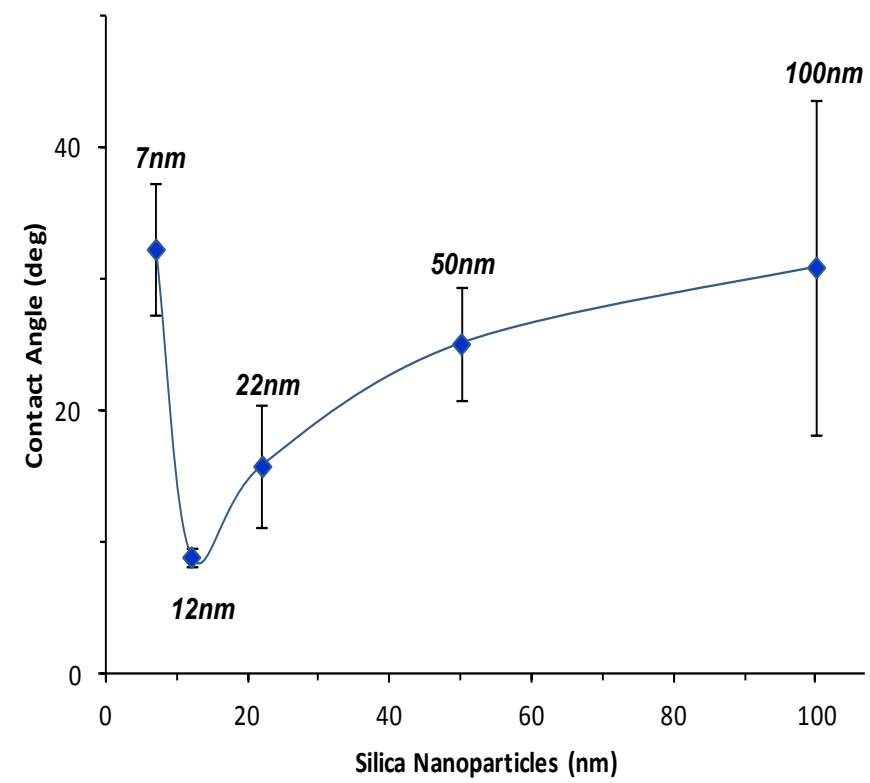

Figure 3: Variation of contact angle on silica nanoparticle sizes with DI water droplets.

In order to confirm the silica nano-assembly, energy dispersive X-ray spectroscopy (EDS) analysis was performed over the microchannel area with and without the silica nanoparticles as shown in Figure 5. The signature of silicon and oxygen peaks in EDS spectra were shown at the microchannel area with the spray silica nanoparticles. This clearly showed that the silica film was successfully constructed inside the COC microchannel by the spray LbL nano-assembly of the silica nanoparticles.

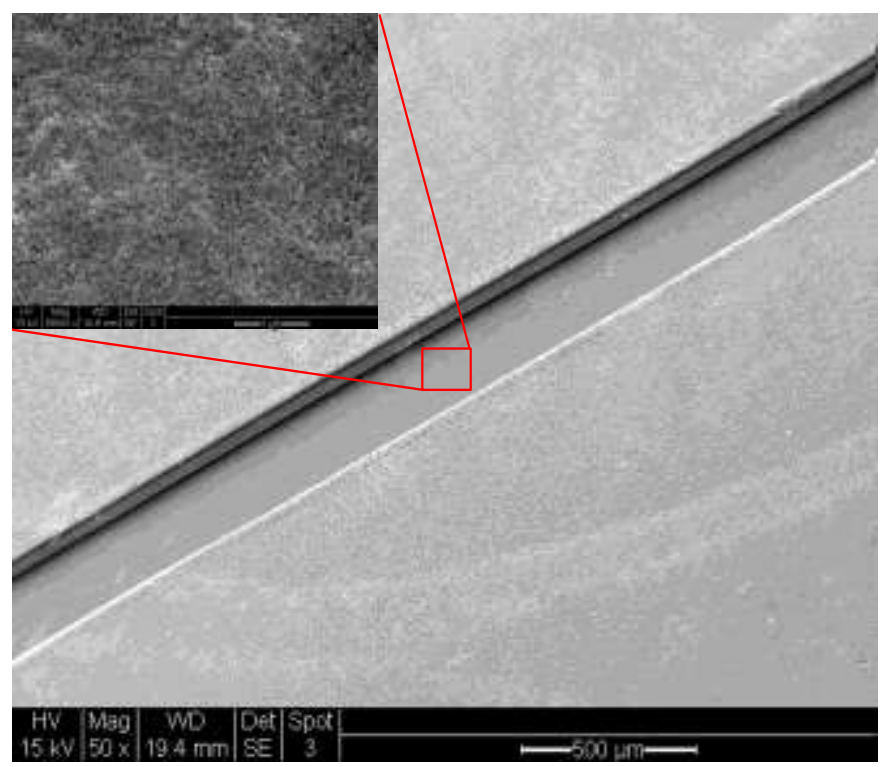

Figure 4: SEM images of COC microchannels after the spray $L b L$ nano-assembly of silica nanoparticles. 


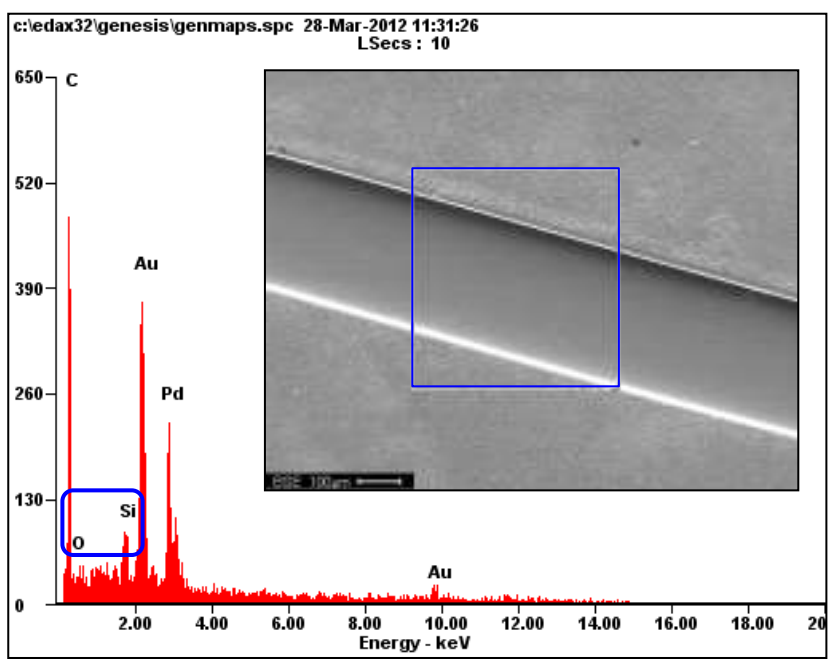

(a)

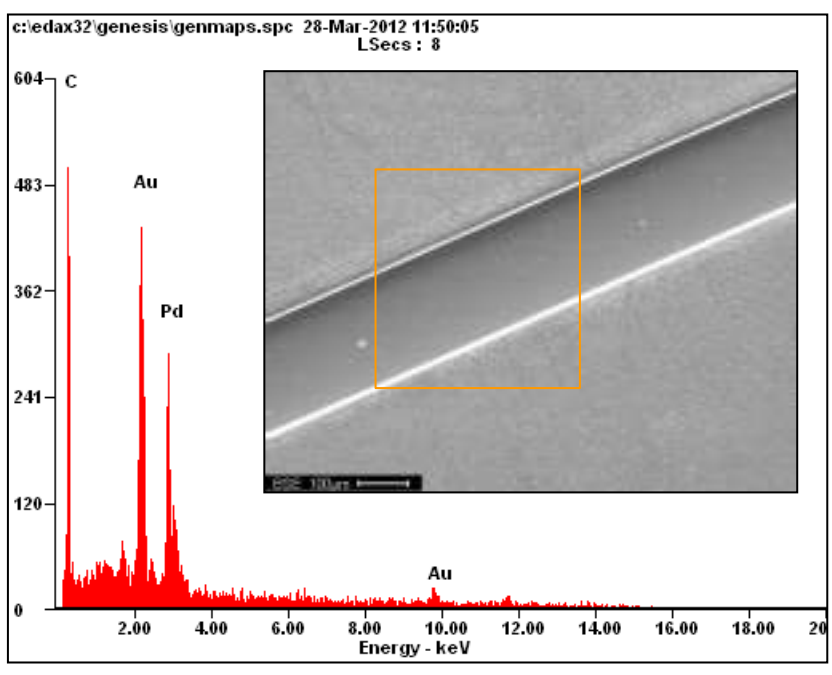

(b)

Figure 5: Energy dispersive X-ray spectroscopy (EDS) spectra of the (a) silica nanoparticle coated COC and (b) bare COC.

\section{Time-dependent Changes in Contact Angle}

Figure 6 shows the changes in contact angle for a first water drop $(3 \mu \mathrm{L})$ as a function of time. The nanoporous multilayer thin films were deposited from the spray LbL assembled silica nanoparticles and a polycation (PDDA) solution. All processing conditions such as spraying time and polyelectrolyte concentration were based on the previous work [11]. The contact angles of all surfaces were decreased over the time. Highly wettable surfaces were obtained with 5,10 and 15 coatings of bilayers, but superhydrophilic behavior was only achieved in 5 coatings of bilayers for the film that completely wets (contact angle below $5^{\circ}$ ) with DI water after 350 seconds. This dramatic drop in contact angles indicates that the COC surface can be super-hydrophilic with silica nanoparticles due to the intrinsically high level of wettability of the silica nanoparticles coupled with the nanoporous nature of the multilayer surface. Thus, the 5 coatings of [PDDA/silica] bilayers were chosen as optimum coatings of bilayers to obtain the maximum nanoporous capillary pumping effect in this process.

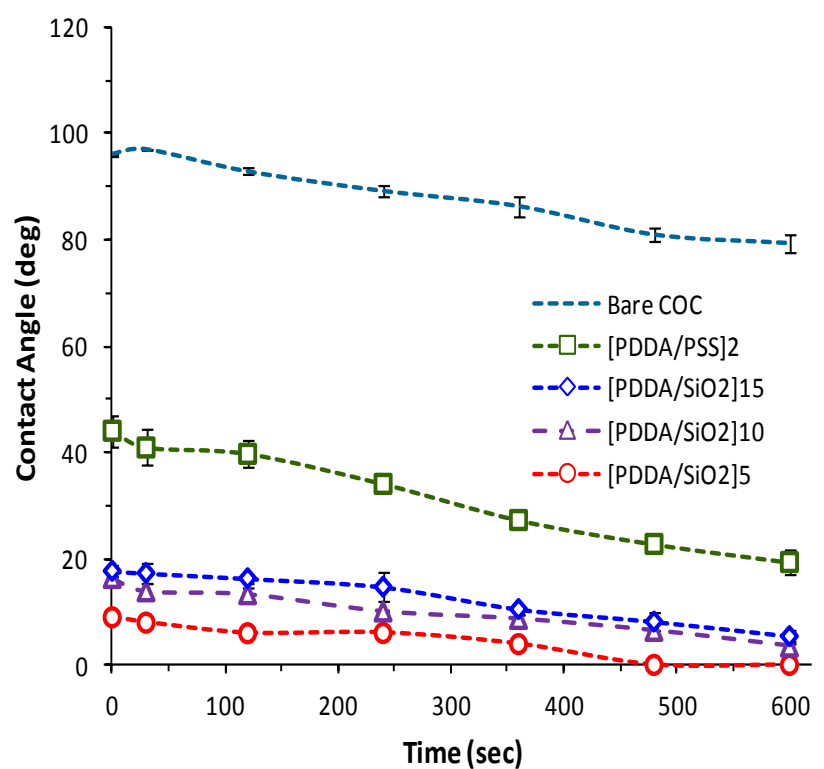

Figure 6: Time-dependent changes in contact angle with the different number of silica layers.

\section{Measurement of Capillary Flow}

Two types of microchannels 200 and $400 \mu \mathrm{m}$ wide in horizontal platform were prepared to characterize the nanoporous capillary pumping effect. All microchannel surfaces were coated with 5 number of [PDDA/silica] bilayers. A droplet of $3 \mu \mathrm{l}$ of red food dye was placed on the inlet of the microchannel using a micropipette. A flow was produced through the microchannel to reach the outlet reservoir by autonomous capillary. As shown in Figure 7, the liquid front of the food dye can move up to $47 \mathrm{~mm}$ without any additional forces within approximately 12 seconds. It is found that the liquid moves quickly at the beginning time, and the speed of the movement slightly decreases at the succeeding time until the liquid reached the outlet reservoir.

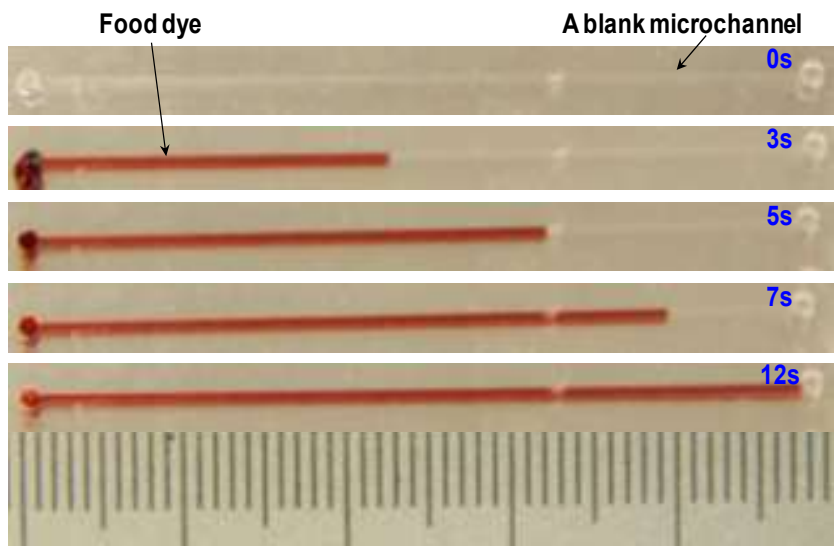

Figure 7: Flow tests for the COC microchannels with a dimension of $400 \mu \mathrm{m} \times 100 \mu \mathrm{m} \times 47 \mathrm{~mm}$. 
Figure 8 shows the relationship between the relative distance of liquid-gas interface locations from inlet to outlet reservoirs and the time. The liquid front location of the food dye moved approximately $42 \mathrm{~mm}$ for the initial seven seconds, and move 5 $\mathrm{mm}$ during the succeeding three seconds when the width of microchannel is $200 \mu \mathrm{m}$. As the width is $400 \mu \mathrm{m}$, the liquid-gas interface location could only move $37 \mathrm{~mm}$ for the first seven seconds, but moves $6 \mathrm{~mm}$ during the succeeding three seconds. Thus, the capillary pumping effect was decreasing with the increasing width of microchannels.

\section{CONCLUSION}

In conclusion, the spray LbL nano-assembly technique creates the nanoporous infiltration networks with silica nanoparticles on the patterned COC microchannels. The nanocapillary effect of the networks, which was confirmed from the measured contact angles, ensures the superhydrophilic surfaces developed on the hydrophobic COC microchannels. The effects were successfully characterized in a horizontal microchannel on COC testing platform. The result shows that this capillary pump can drive the food dye $47 \mathrm{~mm}$ long without additional power within 12 seconds. The nanocapillary driven coating networks can be a general platform for the highly desirable modification of various surfaces of polymer microchannels.

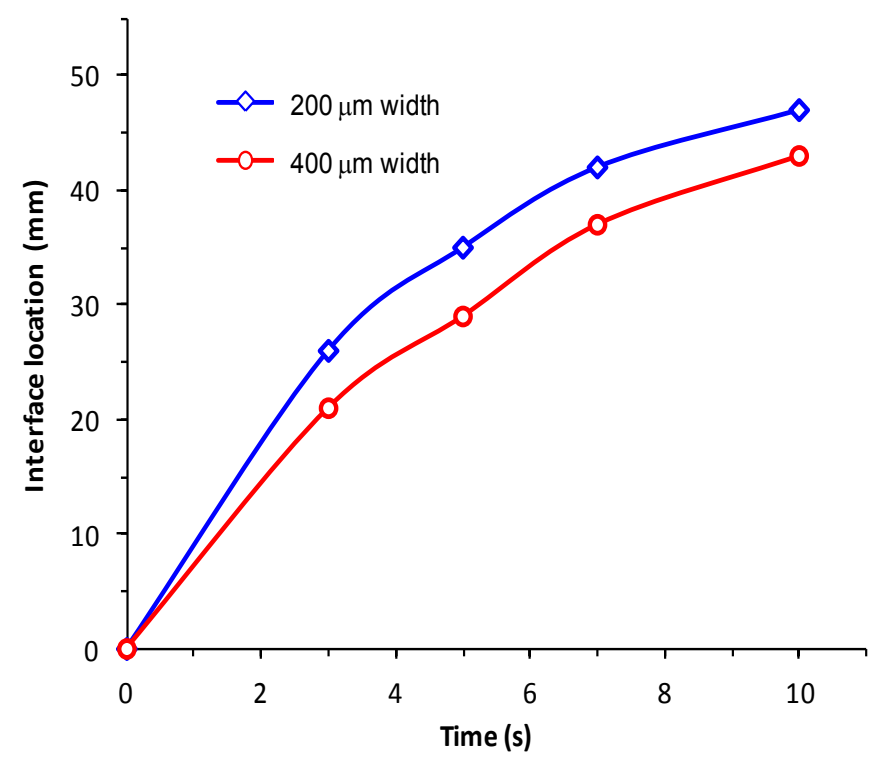

Figure 8: Capillary pumping rate versus time with two types of microchannels 200 and $400 \mu \mathrm{m}$ wide in horizontal platform.

\section{REFERENCES}

[1] C.F. Kung, C.F. Chiu, C.F. Chen, C.C. Chu, "Blood flow driven by surface tension in a microchannel," Microfluidics and Nanofluidics, 6, 693 (2009).

[2] A.C. Henry, T.J. Tutt, M. Galloway, Y.Y. Davidson, C.S. McWhorter, S.A. Soper, R.L. McCarley, "Surface modifcation of poly(methyl methacrylate) used in the fabrication of microanalytical devices," Analytical Chemistry, 72, 5331 (2000).

[3] V. Bulmus, H. Ayhan, E. Piskin, "Modified PMMA monosize microbeads for glucose oxidase immobilization," Chemical
Engineering Journal, 65, 71 (1997).

[4] M.A. Burns, B.N. Johnson, S.N. Brahmasandra, K. Handique, J.R. Webster, M. Krishnan, T.S. Sammarco, P.M. Man, D. Jones, D. Heldsinger, C.H. Mastrangelo, D.T. Burke, "An Integrated nanoliter DNA analysis Device," Science, 282, 484 (1998).

[5] R.R. Lamonte, D. McNally, "Uses and processing of cyclic olefin copolymers," Plastics Engineering, 56, 51 (2000).

[6] J.K. Liu, M.L. Lee, "Permanent surface modification of polymeric capillary electrophoresis microchips for protein and peptide analysis," Electrophoresis, 27, 3533 (2006).

[7] V. Dolnik, "Wall coating for capillary electrophoresis on microchips," Electrophoresis, 25, 3589 (2004).

[8] D. Belder, M. Ludwig, "Surface modification in microchip electrophoresis," Electrophoresis, 24, 3595 (2003).

[9] H. Makamba, J. H. Kim, K. Lim, N. Park, J. H. Hahn, "Surface modification of poly(dimethylsiloxane) microchannels," Electrophoresis, 24, 3607 (2003).

[10] C.H. Ahn, J.W. Choi, G. Beaucage, J.H. Nevin, J.B. Lee, A. Puntambekar, J.Y. Lee, "Disposable Smart Lab on a Chip for Point-of-Care Clinical Diagnostics," Proceedings of the IEEE, 92(1), 54 (2004)

[11] A. Izquierdo, S.S. Ono, J.C. Voegel, P. Schaaf, G. Decher, Langmuir, "Dipping versus Spraying: Exploring the Deposition Conditions for Speeding Up Layer-by-Layer Assembly," Langmuir, 21, 7558 (2005).

\section{CONTACT}

*K.K. Lee, tel: +1-513-535-9030; Leek8@mail.uc.edu 